\title{
FlexQuest como Promotora de Aprendizagem Significativa no Ensino das Ciências Ambientais na Educação Básica
}

\author{
José Antônio Bezerra de Oliveira1, Kátia Aparecida da Silva Aquino2 \\ 1Escola de Referência em Ensino Médio Professora Eurídice Cadaval - Secretaria de \\ Educação e Esportes de Pernambuco (SEE-PE) \\ CEP: 53.700-000 - Itapissuma - PE - Brasil \\ 2Colégio de Aplicação - Universidade Federal de Pernambuco (CAp-UFPE) \\ j.antoniobezerraegmail.com, aquino@ufpe.br
}

\begin{abstract}
This study refers to the creation and validation of a FlexQuest: a tool that combines digital technology with the Theory of Cognitive Flexibility with the purpose of promoting a meaningful learning in students of Basic Education. In this sense the elements promoting knowledge flexibilization were designed and configured a Water Theme FlexQuest. An analysis of conceptual maps constructed by the students was carried out to demonstrate flexible, meaningful and critical learning in progress, promoted through the use of FlexQuest Water. The results show that the tool promoted such levels of learning.
\end{abstract}

Resumo. Este estudo remete à criação e validação de uma FlexQuest: ferramenta que conjuga a tecnologia digital à Teoria da Flexibilidade Cognitiva, com a finalidade de promover uma aprendizagem significativa em estudantes da Educação Básica. Nesse sentido, os elementos promotores de flexibilização do conhecimento foram idealizados e configuraram uma FlexQuest com tema Água. Uma análise de mapas conceituais construídos pelos estudantes foi realizada para evidenciar uma aprendizagem flexível, significativa e crítica em curso, promovida por meio da utilização da FlexQuest Água. Os resultados mostram que a ferramenta promoveu tais níveis de aprendizagem.

\section{Introdução}

Diante da forma como a sociedade vêm se desenvolvendo nos últimos anos, o ambiente e sua biodiversidade acabam por sofrer pela ação indiscriminada do homem (OLIVEIRA, 2006). Nesse cenário, os Parâmetros Curriculares Nacionais (PCN) (BRASIL, 1998) indicam a importância de educar os brasileiros para que ajam de forma sustentável com o ambiente, onde os mesmos se reconheçam como parte integrante do meio. Desta forma, Oliveira (2006) reporta ser a educação o meio mais eficaz para atenuar as problemáticas ambientais atuais, cabendo aos educadores intervirem no processo de ensino e aprendizagem com estratégias que motivem os estudantes a 
compreenderem as dinâmicas ambientais e buscarem alternativas que amenizem os impactos das ações antrópicas no ambiente.

A aprendizagem, a fim de ser efetiva, necessita ser significativa, ou seja, fazer sentido para o estudante, além de desenvolver o pensamento crítico dele, pois não acontece sem a predisposição do mesmo a aprender (MOREIRA 2010, RAMOS e AQUINO 2015). Assim, se desenvolvida de forma crítica, uma aprendizagem significativa faz do estudante um aprendiz díspar, pois ele passa a enxergar o que foi aprendido como uma outra forma de perceber o mundo (MOREIRA 2010).

Nesta direção, o educando disposto a aprender pode atingir a capacidade de reestruturar (desconstruir e reconstruir) um dado conhecimento de diversos modos, dando uma resposta ajustável às situações que ele se depara no dia-a-dia (ALEIXO 2008). Essa dinâmica da reestruturação do conhecimento é descrita pela Teoria da Flexibilidade Cognitiva (TFC), proposta por Spiro e colaboradores nos anos 1980 (WCER 2004). A TFC implica uma aprendizagem flexível, a partir conhecimentos também flexíveis, que formam 'casos' para que o estudante atravesse diversas vezes pelo mesmo assunto, possibilitando a interdisciplinaridade, a multidisciplinaridade e a multidimensionalidade dos conhecimentos, acabando por favorecer a aplicação esses saberes em variados contextos (ALEIXO et al. 2008).

Por outro lado, hoje os estudantes que ingressam na Educação Básica têm, em sua maioria, grande afinidade por tecnologias digitais, sendo receptivos às inovações tecnológicas. Nesse sentido, a utilização de tecnologias da informação e comunicação (TIC) nas aulas pode mudar a concepção do ensino de Ciências: uma abordagem menos conteudista e desinteressante para ser uma prática mais criativa, interativa e motivadora (FRANÇA et al. 2014). A partir do diálogo entre a flexibilização da aprendizagem e o uso de TIC no processo educativo surgiu a FlexQuest (FQ): "recurso didático cujas informações para a aprendizagem partem de casos existentes na internet e não de explicações e interpretações sobre os conteúdos" (VASCONCELOS e LEÃO 2012).

Spiro e colaboradores (1991) apontavam os sistemas hipertexto e hipermídia ideais para a dinâmica da TFC, pois estes sistemas proporcionavam a integração do conhecimento com vários contextos associados (LEÃO et al. 2006). Dessa forma, a FQ é uma ferramenta resultante do aperfeiçoamento de uma WebQuest (WQ) - uma atividade orientada para a pesquisa em que as informações que a compunham advinham da internet. Esta nova configuração da WQ foi idealizada por Souza et al. (2006), apoiados na TFC. A FQ tem base em casos (informações, notícias) retiradas da internet e não de textos estruturados ou finalizados (literais, rígidos) sobre um dado conhecimento. Um caso é descontruído em minicasos, que ilustram diferentes perspectivas do mesmo (LEÃO et al. 2006, ALEIXO et al. 2008). Na FQ também são indicadas conexões entre os minicasos de diferentes casos, e esta interação entre as diferentes perspectivas acabam por trazer diferenciação de significados, reorganização de conhecimentos e possibilita o desenvolvimento de senso crítico nos estudantes por estes observarem aplicabilidade naquilo que está sendo aprendido (SILVA et al. 2015).

A FQ é uma estratégia de promoção de uma aprendizagem flexível e potencialmente significativa conjugada às tecnologias digitais, por seu uso ser possível a partir de dispositivos móveis e internet (LEÃO et al. 2006, ALEIXO et al. 2008, SILVA et al. 2015, OLIVEIRA, SILVA e AQUINO 2017). Assim, o objetivo deste trabalho foi construir e validar uma FQ relacionada à temática ambiental.

\section{Material e Métodos}

\subsection{Construção da FlexQuest}


Com a finalidade de promover a contextualização, a interdisciplinaridade e potencializar a capacidade crítico-reflexiva do estudante acerca do contexto ambiental, esta FQ foi construída na versão 2.0 (Segunda Geração) (OLIVEIRA, GUSMÃO e AQUINO, 2018). O Quadro 1 caracteriza os elementos necessários para a produção de uma FQ de Segunda Geração.

Para favorecer o ensino das Ciências Ambientais, foi escolhida a temática 'Água'. Assim, a FQ foi construída na Plataforma FlexQuest UFRPE (http://www.flexquest.ufrpe.br/), criada pelos pesquisadores da Universidade Federal Rural de Pernambuco para facilitar o manuseio dos elementos utilizados para construção da ferramenta.

Quadro 1. Elementos que integram uma FlexQuest (SANTOS, CLEOPHAS, 2017).

\begin{tabular}{|l|l|}
\hline Contexto & $\begin{array}{l}\text { É exibido o cenário temático, onde pode-se explorar o conhecimento prévio do } \\
\text { estudante para inseri-lo em casos específicos posteriormente. }\end{array}$ \\
\hline Casos & $\begin{array}{l}\text { Ocorre o aprofundamento do tema, de maneira contextualizada e interdisciplinar, } \\
\text { nos casos (com seus minicasos), obtidos na internet e estruturados pelo professor. }\end{array}$ \\
\hline Questões & $\begin{array}{l}\text { Questionamentos onde professor poderá avaliar a evolução conceitual do aprendiz e } \\
\text { verificar se ele consegue analisar, compreender e identificar as relações existentes } \\
\text { entre os contextos dos Recursos. }\end{array}$ \\
\hline Processos & $\begin{array}{l}\text { São conjuntos especiais de minicasos elaborados pelo professor com o propósito de } \\
\text { propiciar ao aluno uma reflexão crítica e flexível entre os diferentes contextos. }\end{array}$ \\
\hline Transferência & $\begin{array}{l}\text { Propõe estimular os aprendizes a continuar o processo começado na FQ, de modo } \\
\text { ativo e crítico, a partir da experiência e dos saberes (re)construídos. }\end{array}$ \\
\hline
\end{tabular}

A seção "Casos" foi composta de quatro casos (eventos/processos relacionados à água) com três ou quatro minicasos (desdobramento destes eventos/processos) cada. Na seção "Questões" foram preparados seis questionamentos a partir das abordagens apresentadas nos Casos. Na seção "Processos" foram organizadas quatro sequências específicas de questionamento. Por fim, na seção "Transferência" foi proposta uma atividade de promoção à aprendizagem ativa, crítica, reflexiva acerca da temática.

\subsection{Validação da FlexQuest}

Como um produto didático, a FQ construída neste estudo necessitou comprovar seu potencial promotor de aprendizagem significativa (OLIVEIRA, SILVA e AQUINO 2017), ou seja, necessitou de validação. Para a validação da FQ um grupo de estudantes $(\mathrm{n}=16)$ do $3^{\circ}$ ano do Ensino Médio da Escola de Referência em Ensino Médio Professora Eurídice Cadaval (Itapissuma, PE, Brasil) foi direcionado a utilizar a ferramenta nas aulas de Biologia (4 horas/aula), formando equipes de quatro pessoas e cumprindo as atividades da FQ descritas no Quadro 2. Estas atividades foram indicadas pelo professor em regência.

Para verificar a capacidade da ferramenta de promover uma potencial aprendizagem com significado e flexível, utilizou-se como estratégia a produção de mapas conceituais individuais pelos estudantes. De acordo com Cañas (2004), estes mapas são formas eficazes de representar a compreensão que um indivíduo possui em relação a um dado conhecimento, evidenciando assimilações, reorganizações e inclusões entre os conceitos presentes na estrutura cognitiva dos estudantes. Sendo assim, antes da aplicação da FQ os estudantes construíram seus mapas de conceitos e, 
após a aplicação da ferramenta, elaboraram novos mapas. Comparações entre os mapas pré e pós-abordagem é que evidenciam se houve uma aprendizagem com significado (OLIVEIRA, SILVA e AQUINO 2017, OLIVEIRA, GUSMÃO e AQUINO 2018).

Quadro 2. Minicasos, questões e atividade de processo escolhidos da FQ para a validação da ferramenta.

\begin{tabular}{|c|l|l|}
\hline Casos & A transposição do Rio São Francisco & $\begin{array}{l}\text { Por que não choveu como deveria no } \\
\text { sertão entre 2011 e 2017? }\end{array}$ \\
\hline Questões & $\begin{array}{l}\text { Reflita sobre sua aptidão para morar no semiárido nordestino que ainda não foi } \\
\text { agraciado pela transposição do São Francisco. Relacionando com o seu atual trato } \\
\text { com a água, escreva quais seriam suas maiores dificuldades e discuta com a sua } \\
\text { equipe. }\end{array}$ \\
\hline Processo & $\begin{array}{l}\text { Revisite os minicasos ('Quem é o Rio São Francisco', 'O que é a transposição' e } \\
\text { 'Por que não choveu como deveria no sertão entre 2011 e 2017?') e, junto com seus } \\
\text { colegas, discuta sobre a situação econômica, ambiental e social dos moradores de } \\
\text { áreas vitimadas pela seca e os efeitos da transposição do Rio São Francisco para } \\
\text { eles. }\end{array}$ \\
\hline
\end{tabular}

\section{Resultados e Discussão}

\subsection{A FlexQuest - Água}

A FQ produzida foi intitulada "Flex-água" (FQ-água) e ela pode ser acessada pelo endereço: http://www.flexquest.ufrpe.br/projeto/6885/geral (Figura 1).

Em um cenário ambiental preocupante, uma atenção especial se volta a um tema presente constantemente no cotidiano dos cidadãos: a água (FREITAS, MARIN, 2015). Bouguerra (2004) reflete a importância da educação para formar indivíduos que tenham uma relação sustentável à água enquanto recurso fundamental, refletindo sobre o uso desta de modo adequado.

O contexto trazido pela FQ-água (Figura 2) insere o estudante em situaçõesproblema que requerem reflexões e ações sustentáveis acerca da água como bem ambiental. Isso auxilia no estabelecimento de pontos da Base Nacional Comum Curricular (BNCC), que direciona o ensino para promover no estudante $o$ desenvolvimento de habilidades de análise, argumentação, debate e avaliação de problemas envolvidos em situações do cotidiano, utilizando conhecimentos das diversas ciências (BRASIL, 2018). 

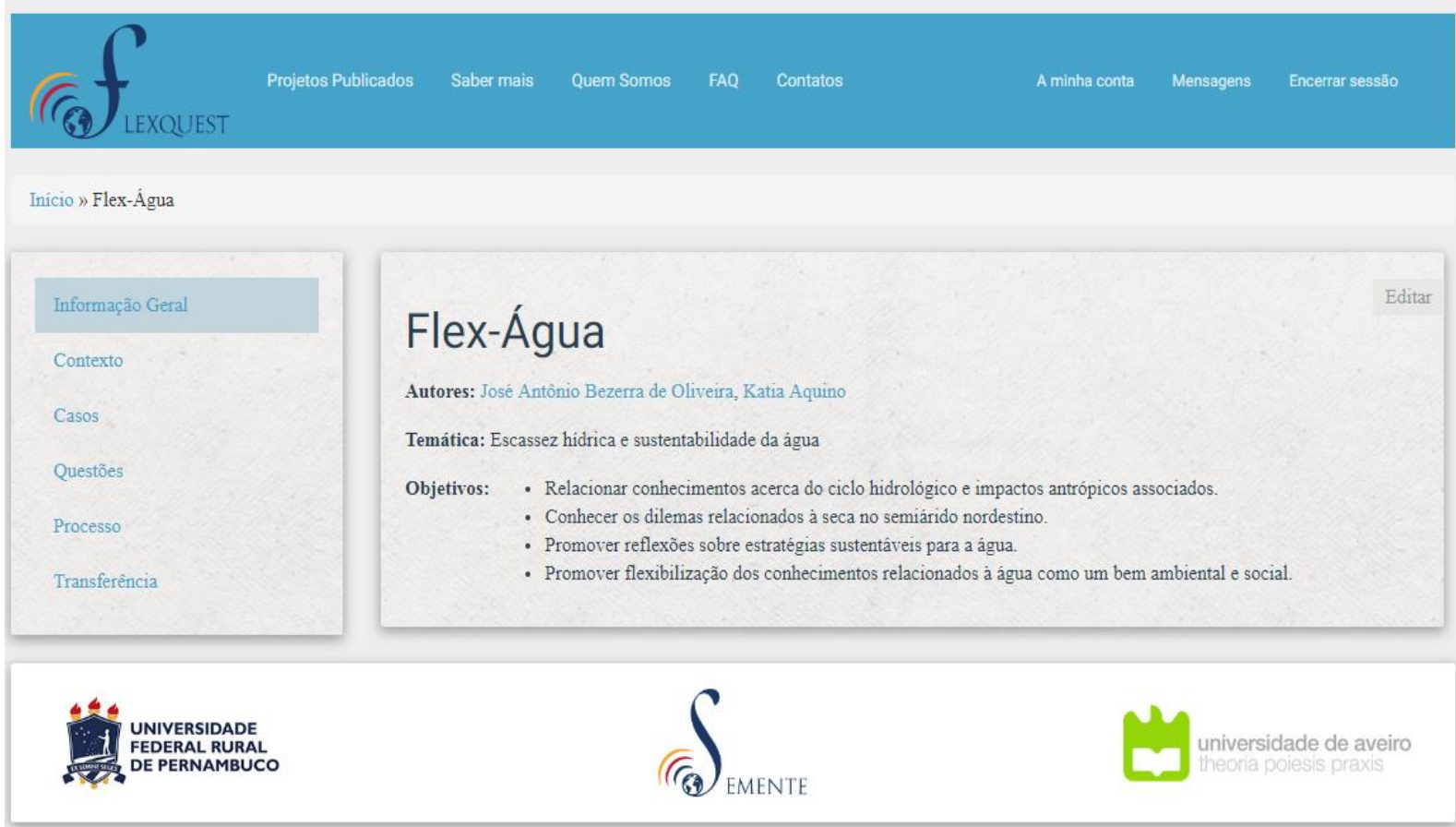

Figura 1. Página inicial da FQ-água.
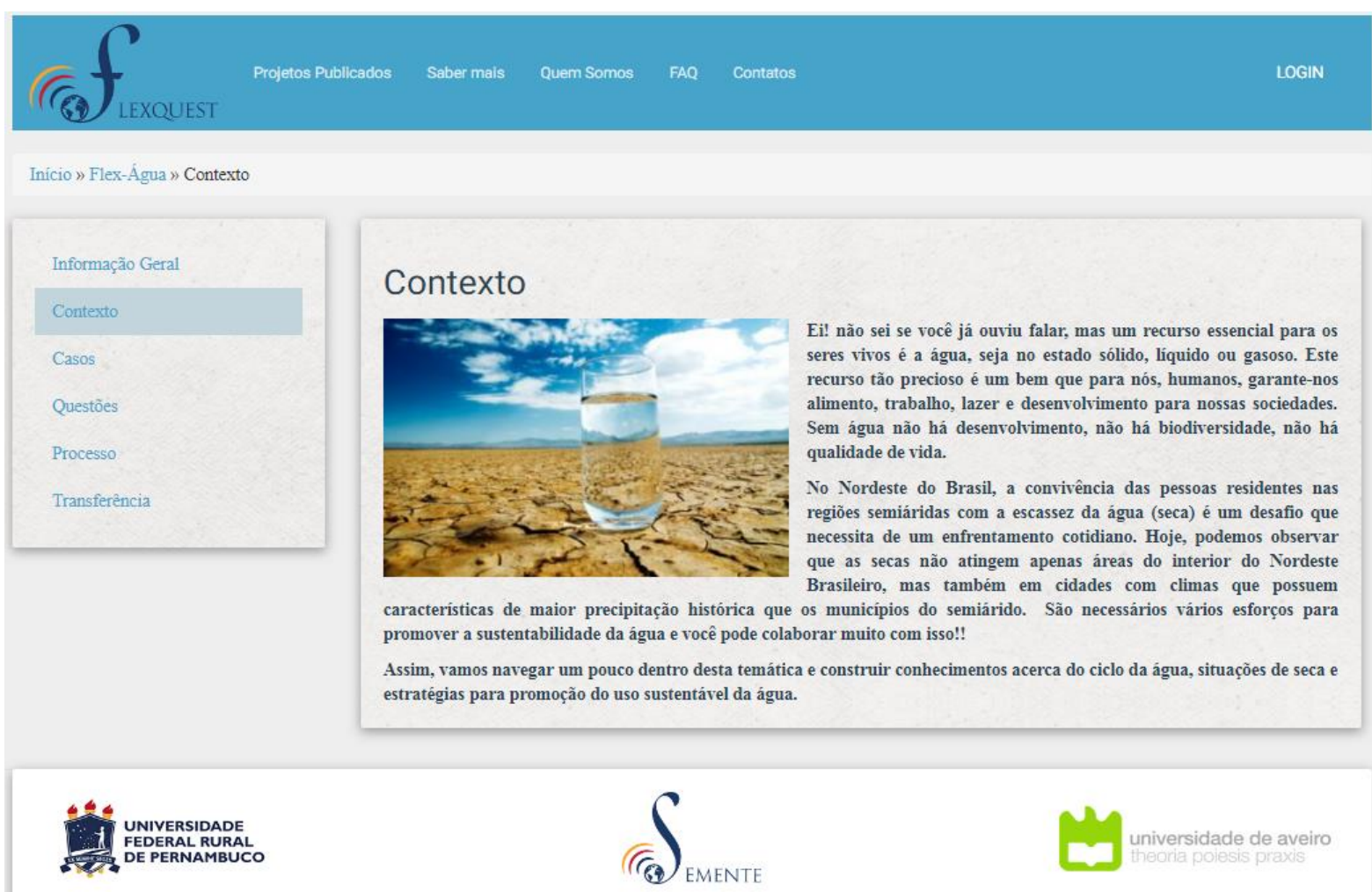

Figura 2. Contexto inicial da FQ-água.

De modo geral, os contextos iniciais presentes nas FQ trazem perguntas ao invés de respostas acabadas e proporcionam a emersão dos conhecimentos prévios dos 
estudantes. Ao situá-los para o contexto do conhecimento, a FQ pode acabar por estimular o interesse do estudante a aprender e esta ação está relacionada, na maioria das vezes, a uma aprendizagem potencialmente significativa e crítica (OLIVEIRA, SILVA e AQUINO 2017, OLIVEIRA, GUSMÃO e AQUINO 2018). Estas reflexões convergem para as considerações de Moreira (2010), o qual afirma que "para ser crítico de algum conhecimento, de algum conceito, primeiramente o indivíduo tem que aprendê-lo significativamente e, para isso, seu conhecimento prévio é, isoladamente, a variável mais importante".

Além da potencial aprendizagem com significado, no cenário da TFC Spiro (1988) aponta que para o desenvolvimento da flexibilidade cognitiva nos estudantes é importante que a estratégia utilizada centre o estudo em casos para o desenvolvimento da conjuntura conceitual do estudante. Esses casos são circunstâncias reais levadas à ambientes de aprendizagem para serem investigadas, extraídas da internet. O Quadro 3 apresenta os casos e seus desdobramentos (minicasos) na construção da FQ-água.

\section{Quadro 3. Casos e seus respectivos minicasos (e seus links de acesso)} utilizados na construção da FQ-água.

\begin{tabular}{|c|c|}
\hline Casos & Minicasos \\
\hline $\begin{array}{l}\text { No Nordeste Brasileiro chove, } \\
\text { mas o sertão é seco } \\
\text { (https://bit.ly/2IPtL1n) }\end{array}$ & $\begin{array}{l}\text { 1. Como acontecem as chuvas no semiárido? } \\
\text { 2. Por que não choveu como deveria no sertão entre } 2011 \text { e } 2017 \text { ? } \\
\text { 3. Como acontecem as chuvas no Litoral do Nordeste }\end{array}$ \\
\hline $\begin{array}{l}\text { A transposição do Rio São } \\
\text { Francisco } \\
\text { (https://bit.ly/2mUCmo8) }\end{array}$ & $\begin{array}{l}\text { 1. Possíveis impactos positivos da transposição } \\
\text { 2. Possíveis impactos negativos da transposição } \\
\text { 3. Quem é o Rio São Francisco? } \\
\text { 4. O que é a transposição? }\end{array}$ \\
\hline $\begin{array}{l}\text { O impacto da ação humana no } \\
\text { ciclo da água } \\
\text { (https://bit.ly/2UODg7x) }\end{array}$ & $\begin{array}{l}\text { 1. Como a água existe em nosso planeta? } \\
\text { 2. O ciclo da água } \\
\text { 3. Ações do homem influenciam no ciclo da água } \\
\text { 4. Como minimizar os impactos da ação do homem no ciclo da } \\
\quad \text { água }\end{array}$ \\
\hline $\begin{array}{l}\text { Reúso de água: uma alternativa } \\
\text { sustentável } \\
\text { (https://bit.ly/2HI5zS6) }\end{array}$ & $\begin{array}{l}\text { 1.Formas de reúso da água } \\
\text { 2.Vantagens do reuso de água residuária } \\
\text { 3.Utilização de água residuária: o que ser? }\end{array}$ \\
\hline
\end{tabular}

Para a seção ‘Questões', os seguintes questionamentos foram propostos:

Questão 1: A água que você toma hoje provavelmente já passou pela goela de algum dinossauro, pode ter vindo do xixi de Isaac Newton, de uma chuva de Londres em 1850 ou até do suor de um cantor famoso do século XX. Reflita e explique como isto é possível.

Questão 2: Reflita sobre sua aptidão para morar no semiárido nordestino que ainda não foi agraciado pela transposição do São Francisco. Relacionando com o seu atual trato com a água, escreva quais seriam suas maiores dificuldades e discuta com a sua equipe.

Questão 3: As plantas são grandes aliadas na conservação de cursos d'água e na formação de chuvas. Pense com sua equipe os porquês desta afirmação estar correta e relacione-os com os eventos de desmatamento.

Questão 4: As variações climáticas de um outro continente ou dos oceanos (Atlântico e Pacífico) influenciam no regime de chuvas do Brasil. Argumente se é correto ou não afirmar que o Brasil pode resolver sozinho o problema da estiagem em suas regiões atingidas. 
Questão 5: As nossas ações podem ser vistas como "atitudes de vida" ou "atitudes de morte". Escolha qual 'atitude' você classificaria as ações de construção de barragens e transposição do Rio São Francisco. Explique os motivos que lhe levaram a classificar assim.

Questão 6: Além de reaproveitar água da chuva, indique outra alternativa viável que possa ser utilizada para aliviar os efeitos da estiagem.

$\mathrm{Na}$ seção 'Processos', as seguintes sequências especiais de questionamento foram construídas:

Sequência 1: Revisite os minicasos ('Quem é o Rio São Francisco', 'O que é a transposição' e 'Por que não choveu como deveria no sertão entre 2011 e 2017?') e, junto com seus colegas, discuta sobre a situação econômica, ambiental e social dos moradores de áreas vitimadas pela seca e os efeitos da transposição do Rio São Francisco para eles.

Sequência 2: Revisite os minicasos ('Vantagens do uso de água residuárias', 'Formas de reúso de água', 'O ciclo da água' e 'Ações do homem influenciam no ciclo da água') e pesquise algum método/estratégia que poderia ajudar no uso sustentável da água.

Sequência 3: A partir dos minicasos ('Ações do homem influenciam no ciclo da água', 'Como minimizar os impactos da ação do homem no ciclo da água?', 'Utilização de água residuária: o que ser?' e 'Formas de reúso da água'), releia-os e aponte como sua comunidade se relaciona com a água e se ela vem cuidando deste recurso de modo sustentável.

Sequência 4: Visite novamente os minicasos ('Utilização de água residuária: o que ser?', 'Ações do homem influenciam no ciclo da água', 'Vantagens do reuso de água residuária' e 'Formas de reúso da água') e pense o que sua equipe poderia fazer para sensibilizar os cidadãos a tomarem atitudes para economia da água.

As Questões propostas na FQ-água se baseiam na reflexão e na investigação dos eventos e notícias indicados nos casos. Dessa forma, o estímulo ao questionamento proposto pela FQ pode levar a um aprendizado por meio de perguntas ao invés de respostas prontas que, segundo Moreira (2010), é um dos princípios para uma aprendizagem crítica e significativa, em que se reflete que o conhecimento não está em cadernos e/ou livros à espera de alguém que venha descobrí-los e compreendê-los, os saberes são construídos a partir da argumentação e emersão de respostas mediante as perguntas.

A atividade de 'Transferência' proposta como prática foi a seguinte: "A partir dos conhecimentos que você construiu ao longo da Flex-água, se reúna com sua equipe e juntos elaborem um livrinho paradidático para as crianças do Ensino Fundamental com algum tema relacionado à água.".

É fundamental que a atividade de transferência organizada pelo professor traga uma situação em que os estudantes possam aplicar os conhecimentos construídos ao longo da FQ e, consequentemente, fazer com que eles compreendam que os conceitos aprendidos não acabam após a utilização da ferramenta. Isso porque eles mesmos podem enfrentar novas situações no cotidiano que exijam ações semelhantes (SANTOS e CLEOPHAS 2017).

\subsection{Validação da FQ-água}

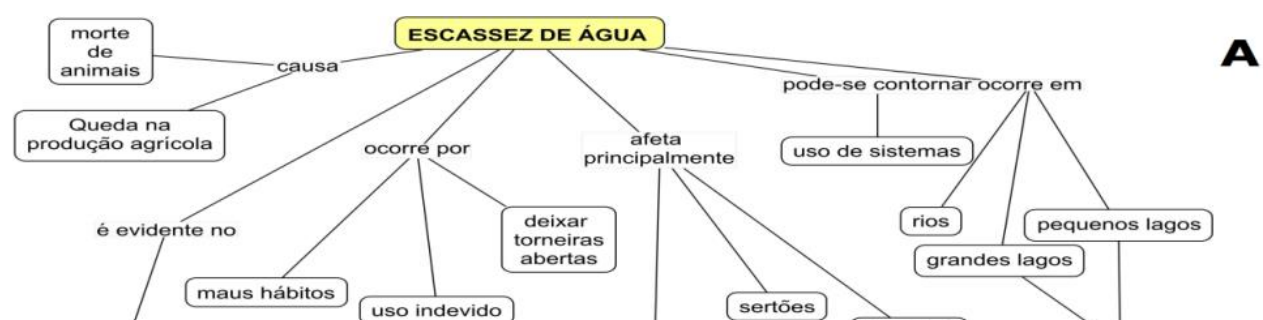


Figura 3. Mapa conceitual inicial (A) e final (B) produzido um grupo de estudantes do Ensino Médio acerca do tema "escassez de água".

Propor aos estudantes a produção de mapas conceituais implica refletir não apenas no resultado gráfico que exibirá algo sobre seu aprendizado, mas principalmente o que fez sentido para eles (OLIVEIRA, SILVA e AQUINO 2017). A Figura 3 indica dois mapas conceituais construídos por um mesmo estudante-alvo da abordagem da FQágua. O primeiro mapa (Figura 2A) mostra as proposições prévias e o segundo mapa (Figura 2B) as proposições estruturadas após a aplicação da FQ. As proposições apresentadas no mapa $\mathrm{B}$ exibem evidências de assimilação de conceitos de um cenário mais complexo do conhecimento. Isso é um dos processos preconizados pela TFC (ALEIXO et al. 2008, SILVA et al. 2015). Ainda no mesmo mapa conceitos presentes nos dois casos apontados para acesso desenvolveram proposições com maior nível de especificação que no mapa inicial, indicando potencial diferenciação progressiva (conceitos amplos sendo especificados). A diferenciação progressiva é um processo relacionado à uma aprendizagem significativa (MOREIRA 2010).

Ainda na interpretação do mapa, é notória certa criticidade que insurge em algumas proposições, como: o Rio São Francisco sendo chamado de Velho Chico (indicando certo sentido de pertencimento), e a relação das doenças que acometem a população do semiárido com a qualidade da água consumida. Uma atitude que chamou a atenção foi o estudante ter determinado a 'solução' entre aspas por, provavelmente, perceber controvérsias no projeto de transposição exibido em um dos casos, o que pode evidenciar um maior grau de criticidade.

Além da análise dos mapas conceituais, em uma discussão posterior à aplicação da FQ-água, o professor de Biologia da turma que passou pelo processo relatou que boa parte dela demonstra argumentos e aponta vários desafios que eles, enquanto cidadãos 
partícipes da sociedade, precisam enfrentar e buscar soluções frente à temática ambiental. Deste modo, percebe-se que as informações da FQ-água auxiliaram os estudantes na elaboração de bons argumentos em que o conhecimento significativo construído ultrapassou os limites da sala de aula para a esfera comunitária, demonstrando, aqui, evidências do desenvolvimento da criticidade dos alunos. Desse modo, corrobora-se com Oliveira et al. (2017), que também observaram estes fenômenos em sua pesquisa envolvendo a construção e validação de uma FQ.

\section{Conclusão}

A partir dos indícios apresentados nos mapas conceituais dos outros estudantes, de modo geral, é possível inferir a FQ-água promoveu uma aprendizagem significativa e com flexibilidade em curso com potencial criticidade para o estudo envolvendo uma temática ligada às Ciências Ambientais, configurando-se como uma ferramenta tecnológica eficaz para o processo de ensino e aprendizagem. A atividade de transferência não foi aplicada, mas o professor-mediador informou que esta etapa da FQ-água será bem interessante no que diz respeito à motivação dos estudantes em executar essa atividade. Eles poderão utilizar os conhecimentos construídos acerca do tema da escassez de água para a construção de um paradidático para crianças do Ensino Fundamental. Esta intencionalidade citada pelo docente remete-nos a uma das premissas básicas de uma aprendizagem que faça sentido para o estudante (MOREIRA 2010, OLIVEIRA, SILVA e AQUINO 2017).

\section{Referências}

ALEIXO, A. A., LEÃO, M. B. C. e SOUZA, F. N. (2008) Flexquest: potencializando a WebQuest no Ensino de Química. R. Faced, 14, 119-133.

BRASIL. (1998) Parâmetros Curriculares Nacionais. Secretaria de Educação Fundamental. Brasília: MEC/SEF, 2000.

FRANÇA, M., SILVEIRA, I. F. e ARAÚJO JR., C. F. (2014) Vivissecção virtual com utilização de tablets: uma contribuição para os processos de ensino e aprendizagem nas aulas de Biologia. In: ARAÚJO-JR. e C. F., SILVEIRA, I. F. (Orgs). Tablets no ensino fundamental e médio: princípios e aplicações, p. 88-92. Terracota.

LEÃO, M. B., SOUZA, F. N., MOREIRA, A. e BARTOLOME, A. R. (2006) FlexQuest: Una Webquest con aportes de la Teoria de la Flexibildad Cognitiva (TFC). Ministerio de Educación de la Naci Libro del Proyecto de Articulacion Universidad Enseñanza Media, 128-143.

MOREIRA, M. A. (2010) Aprendizaje Significativo Critico. Boletín de Estúdios e Investigación. 2 (6) 83-101.

OLIVEIRA, J. A. B., GUSMAO, P. T. R. e AQUINO, K. A. S. (2018) Flexquest como material instrucional potencialmente significativo para o ensino das Ciências Naturais. In: $7^{\circ}$ Encontro Nacional de Aprendizagem Significativa, 2018, Blumenau. Anais do $7^{\circ}$ Encontro Nacional de Aprendizagem Significativa, 7, 105-110.

OLIVEIRA, J. A. B., SILVA, C. J. e AQUINO, K. A. S. (2017) Aprendizagem Significativa Crítica e Flexibilidade Cognitiva: diálogo metodológico através da construção e validação de uma ferramenta Flexquest para o ensino de Ecologia na educação básica. Cadernos de Estudos e Pesquisa na Educação Básica, 3, (1), 35-51. 
OLIVEIRA, N. A. S. (2006) A educação ambiental e a percepção fenomenológica, através de mapas mentais. Revista eletrônica do Mestrado em Educação Ambiental. Rio Grande, 16, 32-46.

RAMOS, P. S. e AQUINO, K. A. S. (2015) Ações na parte diversificada do currículo: um relato de experiência na perspectiva de uma aprendizagem significativa crítica. Cadernos de Estudos e Pesquisa na Educação Básica, 1, 240-249.

SANTOS, I. G. S. e CLEOPHAS, M. G. (2017) Uma proposta de trabalho interdisciplinar sobre a água: o caso da Flexquest "O Fluido da Vida". Enseñanza de las ciencias, n. Extra, 4949-4954, 2017.

SILVA, I. G. S. S., LEÃO, M. B. C. e SOUZA, F. N. (2015) Plataforma Flexquest $\AA_{\text {: }}$ Uma estratégia didática para a promoção de flexibilidade cognitiva e interdisciplinaridade com recursos Web 2.0. Revista Ibérica de Sistemas e Tecnologias de Informação, SPE4, 35-49.

SOUZA, F. N. e LEÃO, M. B. C. (2006) MOREIRA, A. Elementos estruturadores de uma WebQuest flexível (FlexQuest). In: Encontro sobre Webquest. p. 223-227, Universidade de Minho.

VASCONCELOS, F. C. G. C. e LEÃO, M. B. C. (2016) Utilização de recursos audiovisuais em uma estratégia Flexquest sobre radioatividade. Investigações em Ensino de Ciências, 17(1), 37-58.

WCER (2004) Cognitive flexibility. http://www.wcer.wisc.edu/step/edpsych301/document/CognitiveFlexibility.htm, Janeiro. 